\title{
Variabilidade espacial das frações granulométricas e da salinidade em um Neossolo Flúvico do semi-árido
}

\author{
Spatial variability of texture and salinity in a Fluvic Neossol of the semi-arid \\ Edivan Rodrigues de Souza' Abelardo Antônio de Assunção Montenegro ${ }^{I I}$ \\ Suzana Maria Gico Lima Montenegro ${ }^{\text {III }}$ Thaís Emanuelle Monteiro dos Santos ${ }^{\text {II }}$ \\ Tafnes da Silva Andrade ${ }^{\mathrm{II}}$ Elvira Régis Pedrosa ${ }^{\mathrm{II}}$
}

\section{RESUMO}

A análise dos atributos físicos e da salinidade de solos em áreas irrigadas é de extrema importância para elaboração e implantação de propostas de manejo. Com o objetivo de avaliar a dependência espacial das frações granulométricas e da condutividade elétrica do extrato de saturação, foi desenvolvido um estudo em área aluvial irrigada no semi-árido de Pernambuco, em que se adotou uma malha regular de $5 \times 5 \mathrm{~m}$, correspondendo a um total de 98 pontos amostrais, e foram coletadas amostras de solo na profundidade de $0-20 \mathrm{~cm}$. As variáveis foram submetidas à análise estatística clássica, seguida de análise geoestatística. A variabilidade dos dados foi classificada como média para todas as variáveis estudadas, com coeficientes de variação respectivamente de 26,$08 ; 14,81 ; 16,01$ e 28,00\% para areia, argila, silte $e$ condutividade elétrica do extrato de saturação. A dependência espacial foi considerada alta para as frações granulométricas e moderada para a condutividade elétrica, com alcances de 56, 50, 55 e 35m, respectivamente. A condutividade elétrica está fortemente correlacionada espacialmente com o teor de silte, apresentando alcance de $52 \mathrm{~m}$ para o semivariograma cruzado. Os resultados ressaltam a importância da fração silte na distribuição espacial da salinidade, bem como na relevância para o manejo racional do solo estudado.

Palavras-chave: geoestatística, condutividade elétrica, solos francos.

\section{ABSTRACT}

Analysis of the physical attributes and salinity of soils is important for proposing management strategies in irrigated areas. The objective of this study was to evaluate the spatial dependence of the granulometric fractions and electric conductivity of the saturation extract in an area irrigated in the semi-arid of Pernambuco State. A $5 \times 5 \mathrm{~m}$ regular mesh was adopted, with 98 sampling points collected at depth $0-20 \mathrm{~cm}$. The variables have been analysed through statistics and geoestatistics. The variation coefficients of the saturation extract were $26.08 ; 14.81 ; 16.01$ and $28.00 \%$ for sand, clay, silt and electrical conductivity of the saturation extract, respectively. The spatial dependence was identified as high for the granulometric fractions and as moderate for the electric conductivity, with ranges of 56; 50 and 55 and 35m, respectively. The electric conductivity has a strong spatial correlation with silt content, presenting range of $52 \mathrm{~m}$, for the cross semivariogram. The results highlight the importance of the silt fraction in the spatial distribution of salinity in loam soils, and its relevance for adequate management of these soils.

Key words: geoestatistics, electric condutivity, loam soils.

\section{INTRODUÇÃO}

Estudos que enfocam a variabilidade espacial de propriedades físicas, químicas e hidráulicas de solos têm grande relevância na literatura, principalmente como subsídio para o manejo racional de água e solo em perímetros irrigados. Tais variações espaciais são decorrentes das taxas variáveis nas quais atuam os processos de formação e das diversas atuações antrópicas durante seu cultivo (CICHOTA et al. 2003; REICHARDT \& TIMM, 2004). No que diz respeito a atributos físicos, EGUCHI et al. (2002) comentam que a textura e a densidade de partículas são consideradas elementos de grande importância na identificação e na classificação do solo, pois se

'Departamento de Agronomia, Universidade Federal Rural de Pernambuco (UFRPE), 52171-900, Recife, PE, Brasil. E-mail: edivanrs@hotmail.com. Autor para correspondência.

"Departamento de Tecnologia Rural, UFRPE, Recife, PE, Brasil.

IIIDepartamento de Engenharia Civil, UFPE, Recife, PE, Brasil. 
constituem em características físicas de elevada estabilidade. De acordo com JOURNEL \& HUIJBREGTS (1978), a variação espacial de atributos físicos do solo não ocorre de modo aleatório, mas sim segundo uma estrutura de dependência espacial, que deve ser investigada e incorporada aos processos de mapeamento. Segundo COELHO (1983), com exceção do $\mathrm{pH}$ dos solos, as demais propriedades químicas podem apresentar variação acentuada, o que justifica o estudo destas propriedades através de técnicas geoestatísticas. Dentre os autores que realizam pesquisas com esse enfoque, pode ser destacado o trabalho de SOUZA et al. (2000), que estudaram a variabilidade espacial da salinidade de um solo aluvial no semi-árido paraibano com intuito de realizar um manejo mais eficiente do solo. Dentre os vários tipos de solo, os aluviais são bastante utilizados nas atividades agrícolas, por isso há necessidade de pesquisas que proporcionem melhor avaliação de sua variabilidade espacial, visando à adoção de técnicas adequadas de manejo agrícola (SOUZA et al., 2000).

O objetivo deste trabalho foi examinar a dependência espacial das frações granulométricas do solo e da condutividade elétrica do extrato de saturação de um solo aluvial sob cultivo agrícola em um lote aluvial irrigado, a fim de investigar a dependência espacial entre as frações e a salinidade de modo a orientar ações de manejo e amostragem mais eficientes.

\section{MATERIAL E MÉTODOS}

A área objeto do estudo está localizada na sub-bacia do Rio Ipanema, no Estado de Pernambuco, com área de $194,82 \mathrm{~km}^{2}$. A área está localizada entre as coordenadas $8^{\circ} 34$ ' 17 ' e $8^{\circ} 18^{\prime} 11$ ”' de latitude sul, e $37^{\circ}$ 1'20" de longitude oeste, com altitude média de $613 \mathrm{~m}$. Este domínio insere-se em vale aluvial do Riacho Mimoso, em particular na Fazenda Nossa Senhora do Rosário, com solo predominantemente caracterizado como Neossolo Flúvico (BASTOS, 2004). De acordo com a classificação de Köppen, o clima na região é do tipo BSsh (extremamente quente, semi-árido), com precipitação total anual média de $730 \mathrm{~mm}$ e evapotranspiração potencial anual média de 1683mm (HARGREAVES, 1974).

Foi adotada área irrigada piloto com dimensão de $1950 \mathrm{~m}^{2}$ para análise espacial. O solo amostrado vem sendo explorado aproximadamente há dezesseis anos, principalmente com o cultivo de hortaliças, dentre elas, pimentão, repolho e cenoura. O cultivo intensivo é irrigado a partir de poço amazonas, que apesar de apresentar teor elevado de sais (condutividade elétrica média anual de 1,3dS m-1), vem apresentando produtividade satisfatória, graças às técnicas de manejo advindas de profissionais inseridos em projetos de pesquisa que prestam assessoria aos agricultores.

A realização da coleta do solo ocorreu no mês de agosto de 2005, logo após o período chuvoso da região. O solo foi amostrado após a preparação para o cultivo da cenoura, o qual foi submetido à aração e à gradagem. As amostras foram coletadas na profundidade de $0-20 \mathrm{~cm}$ através de um trado holandês. Visando caracterizar a dependência espacial, foi estabelecida uma malha regular de $5 \times 5 \mathrm{~m}$, inserida em um retângulo de 65 x 30m, totalizando 98 pontos amostrados. As amostras foram secas ao ar, destorroadas, e passadas em peneira de malha de 2,0 $0 \mathrm{~mm}$. As percentagens de areia, argila e silte foram determinadas obedecendo ao método do densímetro (EMBRAPA, 1997). Já o preparo e a extração da pasta saturada do solo foram realizados de acordo com RICHARDS (1954). Após a extração, foi determinada a condutividade elétrica do extrato de saturação (CEes) através de condutivímetro digital.

Com o objetivo de verificar o comportamento dos dados, foi realizada análise estatística descritiva, através da avaliação de medidas de tendência central (média, mediana e moda) e dispersão (desvio padrão, variância, coeficiente de variação) e a aderência à distribuição normal, segundo o teste de Kolmogorov-Smirnov, aos níveis de 1 e 5\% de significância. Para análise geoestatística, utilizouse a ferramenta geoestatística GEO-EAS (ENGLUND \& SPARKS, 1991), adotando-se o estimador clássico da semivariância (JOURNEL \& HUIJBREGTS, 1978). Adicionalmente, foi verificado o desempenho do estimador “robusto" proposto por CRESSIE \& HAWKINS (1980), o qual é compatível com a presença de certo grau de discrepância nos dados em relação à distribuição normal, conforme discutido em MONTENEGRO et al. (1999). Após a obtenção dos semivariogramas experimentais, foram testados os modelos gaussiano, esférico e exponencial e foi escolhido aquele que apresentou ajuste adequado aos valores experimentais e erros padronizados com média próxima a zero e desvio padrão próximo à unidade, segundo o critério de Jack-Knifing (VAUCLIN et al., 1983).

Para análise da correlação espacial entre duas variáveis distribuídas, utilizou-se o semivariograma cruzado, segundo funcional proposta por VAUCLIN et al. (1983).

Para caracterização do grau de variabilidade, foram analisados os valores de coeficiente de variação (CV) dos atributos, conforme sugerido por WARRICK 
(1998), o qual considera baixa variabilidade quando CV < 15\%; média para o intervalo 15 - 50\%, e alta variabilidade quando $\mathrm{CV}>50 \%$.

Os dados discrepantes foram eliminados com base no critério de HOAGLIN et al. (1983), que consideram discrepantes aqueles dados abaixo do limite inferior ( $\mathrm{Li}$ ) ou acima do limite superior (Ls) respectivamente, estimados por: $\mathrm{Li}=\mathrm{Qi}-1,5 \mathrm{Ai}$ e Ls = Qs + 1,5Ai, sendo Qi e Qs os quartis inferior e superior, respectivamente, e Ai a amplitude interquartílica.

No tocante ao grau de dependência espacial, utilizou-se a classificação proposta por CAMBARDELLA et al. (1994), na qual um determinado atributo pode ser considerado como exibindo forte, moderada ou fraca dependência espacial, em função da relação entre o efeito pepita e o patamar de seu semivariograma ajustado. Valores inferiores a $25 \%$ caracterizam forte dependência espacial, entre $25 \%$ e $75 \%$ moderada, enquanto que acima de $75 \%$ fraca dependência. No tocante à confecção dos mapas de isolinhas, utilizando o algoritmo da krigagem, foi adotado o programa computacional Surfer for Windows versão 8.0 (SURFER, 2002).

\section{RESULTADOS E DISCUSSÃO}

Os coeficientes de curtose e assimetria mostram que é possível assumir que os dados seguem distribuição normal de probabilidade (Tabela 1). Com efeito, o coeficiente de assimetria deve se aproximar de 0 , enquanto o de curtose deve ser próximo de 3 . Nota- se que, para todas as variáveis analisadas, os valores de tais coeficientes se aproximaram dos padrões requeridos. O teste de Kolgomorov Smirnov indicou normalidade dos dados ao nível de 1\% de significância para a areia, a argila e o silte, e de 5\% para a CEes.

Na tabela 1 , pode-se verificar que as variáveis estudadas neste trabalho se caracterizam como de média variabilidade, segundo o critério de WARRICK (1998), exceto a fração argila que se caracteriza como de baixa variabilidade. SOUZA et al. (2000), trabalhando em solo aluvial no Estado da Paraíba, verificaram elevada variação para CEes (CV entre 135,85 e 173,15\%) em todas as profundidades estudadas. Esses pesquisadores atribuem a variabilidade encontrada à heterogeneidade, como resultado dos processos de formação inerentes a esses solos, que ocorrem pela acumulação e distribuição desuniformes de sucessivos depósitos de materiais oriundos de outros lugares, proporcionados pela posição topográfica que ocupam, além do manejo a que são submetidos, entre outros fatores. Já MONTENEGRO \& MONTENEGRO (2006), pesquisando em um transecto na mesma área de estudo deste trabalho, encontraram um CV de $118 \%$ para a CEes. Percebe-se através das médias das frações granulométricas presentes na tabela 1 e pelo conjunto original de dados que o solo apresenta uma classe textural predominantemente franca.

Após a verificação da estatística descritiva dos dados, confeccionou-se os semivariogramas experimentais, como também se procurou ajustar

Tabela 1 - Estatística descritiva para as frações granulométricas e condutividade elétrica do extrato de saturação (CEes) e parâmetros dos modelos de semivariogramas ajustados aos dados experimentais.

\begin{tabular}{|c|c|c|c|c|}
\hline \multirow{2}{*}{ Estatística descritiva } & \multicolumn{3}{|c|}{--------------Frações granulométricas (g kg ${ }^{-1}$ )-------------- } & \multirow{2}{*}{ CEes $\left(\mathrm{dS} \mathrm{m} \mathrm{m}^{-1}\right)$} \\
\hline & Areia & Argila & Silte & \\
\hline Média & 361,50 & 230,10 & 398,40 & 1,12 \\
\hline Mediana & 340,00 & 230,00 & 420,00 & 1,11 \\
\hline Desvio padrão & 94,28 & 34,10 & 63,80 & 0,32 \\
\hline C V (\%) & 26,08 & 14,81 & 16,01 & 28,00 \\
\hline Assimetria & 0,72 & $-0,29$ & $-0,85$ & 0,51 \\
\hline Curtose & 2,74 & 2,35 & 3,03 & 2,70 \\
\hline Quartil inferior & 280,00 & 210,00 & 360,00 & 0,88 \\
\hline Quartil superior & 420,00 & 260,00 & 450,00 & 1,32 \\
\hline \multirow[t]{2}{*}{ Amplitude Total } & 410,00 & 140,00 & 300,00 & 1,31 \\
\hline & -------- & râmetros do & ivariograma & ---------- \\
\hline Efeito pepita (Co) & 650,00 & 125,00 & 300,00 & 0,07 \\
\hline Patamar (Co + C1) & $9.550,00$ & $1.075,00$ & $4.450,00$ & 0,115 \\
\hline Alcance & 56,00 & 50,00 & 55,00 & 35,00 \\
\hline $\mathrm{Co} / \mathrm{Co}+\mathrm{C}_{1}$ & 6,81 & 11,62 & 6,74 & 61,00 \\
\hline Modelo & Gaussiano & Gaussiano & Gaussiano & Gaussiano \\
\hline
\end{tabular}

$\mathrm{Co} / \mathrm{Co}+\mathrm{C}_{1}$ - grau de dependência espacial de Cambardella (\%); CV - Coeficiente de Variação. 
modelos através dos quais fosse melhor caracterizado o comportamento espacial das variáveis estudadas. As variáveis, os modelos ajustados e os parâmetros dos modelos podem ser visualizados na tabela 1 e na figura 1. O modelo de melhor ajuste, para todas as variáveis, foi do tipo gaussiano.

Os teores de areia, argila e silte se caracterizam por apresentar uma forte dependência espacial, já que apresentaram, conforme CAMBARDELLA et al. (1994), graus de dependência espacial de 6,81; 11,62 e 6,74\% respectivamente (Tabela 1). De acordo com GREGO \& VIEIRA (2005), a ocorrência da dependência espacial na camada superficial pode estar associada ao preparo do solo convencional, onde os equipamentos de preparo (arado e grade) movimentam demasiadamente a camada superior, afetando sua estrutura original, e tornando pontos próximos entre si mais semelhantes que os mais distantes. Os alcances encontrados neste trabalho foram de 56, 50 e 55m para areia, argila e silte respectivamente. Avaliando um transecto de $100 \mathrm{~m}$ com 51 pontos amostrais em solo aluvial, EGUCHI et al. (2002) encontraram alcances de 15; 49,5 e 14,5m para areia, argila e silte, e atribuíram os resultados principalmente às contribuições presentes na formação do solo. Do mesmo modo, VIEIRA (1997) encontrou alcances de $15 \mathrm{~m}$ para argila e silte em um Latossolo Roxo Distrófico sob preparo convencional, em Campinas-SP, trabalhando em uma parcela de $30 \times 30 \mathrm{~m}$, com malha de regular de $5 \mathrm{~m}$ nas duas direções, formando 49 pontos amostrais. Essa diferença pode ser atribuída às características físicas do Latossolo, que diferem bastante do Neossolo Flúvico estudado neste trabalho.

Quando se verifica o comportamento da CEes, percebe-se uma moderada dependência espacial, entre 25 e $75 \%$, segundo critério de CAMBARDELLA et al. (1994), principalmente devido ao alto valor de efeito pepita (Figura 1 e Tabela 1). Isso pode ser atribuído à descontinuidade do semivariograma para distâncias inferiores à menor distância amostrada, bem como a incertezas de laboratório, associadas também à metodologia de determinação desta variável, quando são adotadas características subjetivas para identificar o ponto em que a pasta encontra-se saturada. Resultados semelhantes foram obtidos por FAULIN (2005), utilizando um sensor de condutividade elétrica em campo nas profundidades de $0-30$ e $30-90 \mathrm{~cm}$, em

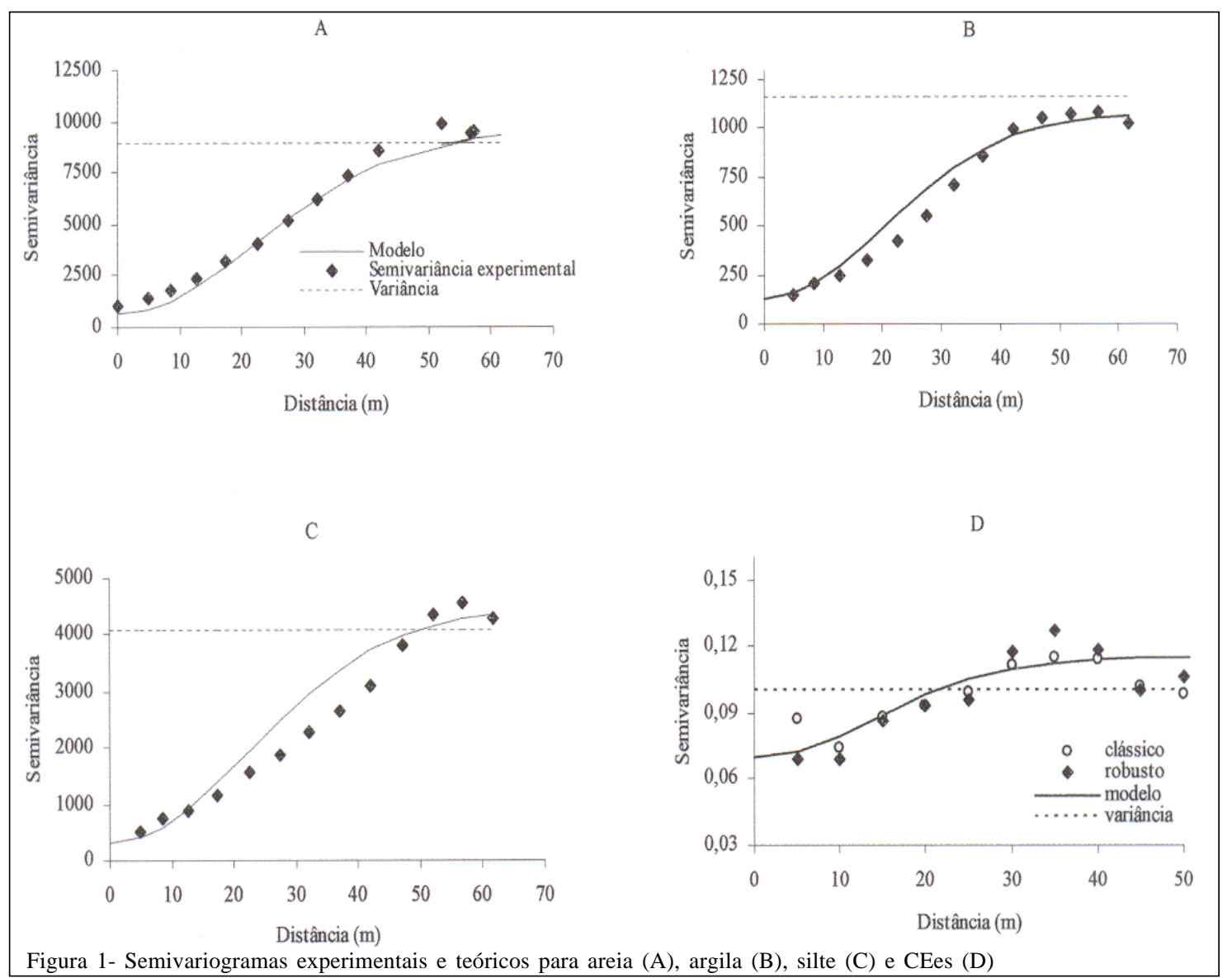

Ciência Rural, v.38, n.2, mar-abr, 2008. 
duas áreas comerciais. Verificou-se que em uma das áreas os semivariogramas caracterizaram-se por apresentar moderada dependência espacial nas duas profundidades avaliadas, tendo-se atribuído esse comportamento às elevadas precipitações antecedentes às medições de CEes. Tal justificativa pode ser adotada também para esclarecer a moderada dependência espacial encontrada para a condutividade elétrica de que trata este trabalho, tendo em vista que as amostras foram coletadas logo após o período chuvoso.

A realização da coleta ocorreu no mês de agosto de 2005, logo após o período chuvoso da região. Vale destacar que durante o mês de dezembro de 2004 a novembro de 2005 foram contabilizados 714,28mm devido às precipitações ocorridas na área de estudo, sendo que $322,86 \mathrm{~mm}$ ocorreram nos meses de abril a julho de 2005. SOUZA et al (2000), trabalhando em solo aluvial afetado por sais, separaram as áreas afetadas e não-afetadas pela salinização e verificaram que, para as áreas não-afetadas, ou seja, normais, não foi encontrada dependência espacial para a profundidade de $0-20 \mathrm{~cm}$. Um fator que pode influenciar estudos de dependência espacial é a escala de amostragem, fato pelo qual MONTENEGRO \& MONTENEGRO (2006), trabalhando em um transecto na mesma região, encontraram alcance de 520,32m para a CE do extrato de saturação em escala de perímetro irrigado.

Na figura 1, para a CEes, as semivariâncias experimentais foram obtidas a partir do estimador clássico, bem como do estimador robusto de Cressie, em que se percebe que para as menores distâncias as semivariâncias são menores, evidenciando a robustez do estimador de Cressie, uma vez que atribui pesos maiores as menores distâncias, em virtude do maior número de pares.

Os semivariogramas ajustados para a CEes e o silte (Figura 1) foram validados através da metodologia de Jack-knifing (VAUCLIN et al., 1983), produzindo respectivamente resíduos com médias 0,07 ; -0,051 e desvios padrão 1,065; 1,063.

Para avaliar a correlação espacial entre o silte e a CEes, foi construído um semivariograma cruzado (Figura 2), no qual se pode verificar a elevada dependência espacial entre tais grandezas. O alcance encontrado foi de 51,96m, próximo dos valores dos alcances obtidos para a CEes e o silte (Tabela 1). Resultados semelhantes foram encontrados por MONTENEGRO \& MONTENEGRO (2006), que obtiveram alcances com mesma ordem de grandeza para o semivariograma da CEes e para o semivariograma cruzado da CEes e silte.

Tal resultado pode ser evidenciado nos mapas produzidos para silte e condutividade elétrica (Figura 3). A ocorrência de maiores condutividades elétricas está associada a regiões apresentando elevados teores de silte. Com efeito, RIBEIRO et al. (2003) discutem que os solos aluviais com maiores teores de silte apresentam predisposição à salinização secundária, proporcionando uma maior deposição de sais na superfície do solo, acarretando o aumento da condutividade elétrica do extrato de saturação.

\section{CONCLUSÕES}

Verifica-se que as variáveis analisadas obedecem à distribuição normal de freqüência e que possuem uma variabilidade média, exceto para fração

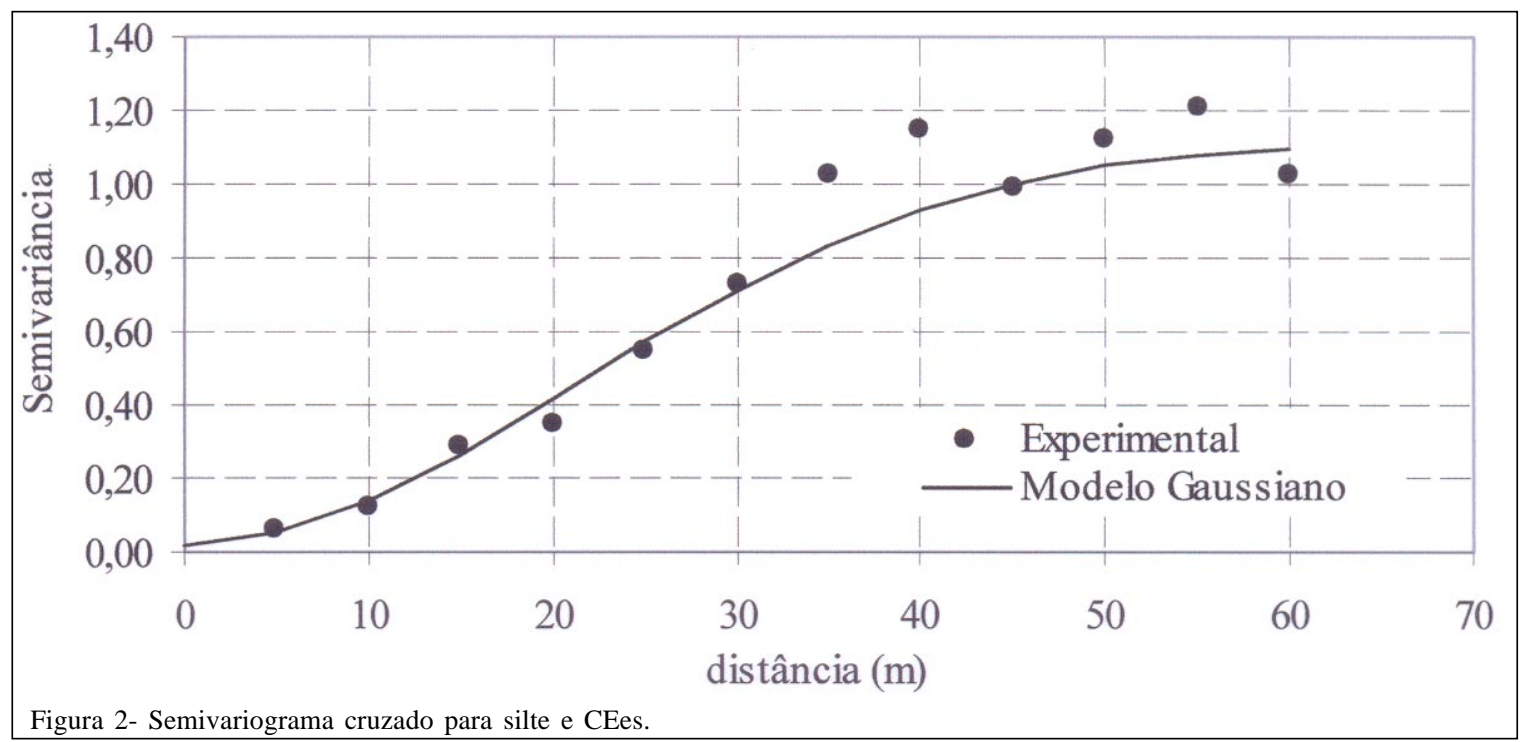

Ciência Rural, v.38, n.2, mar-abr, 2008. 


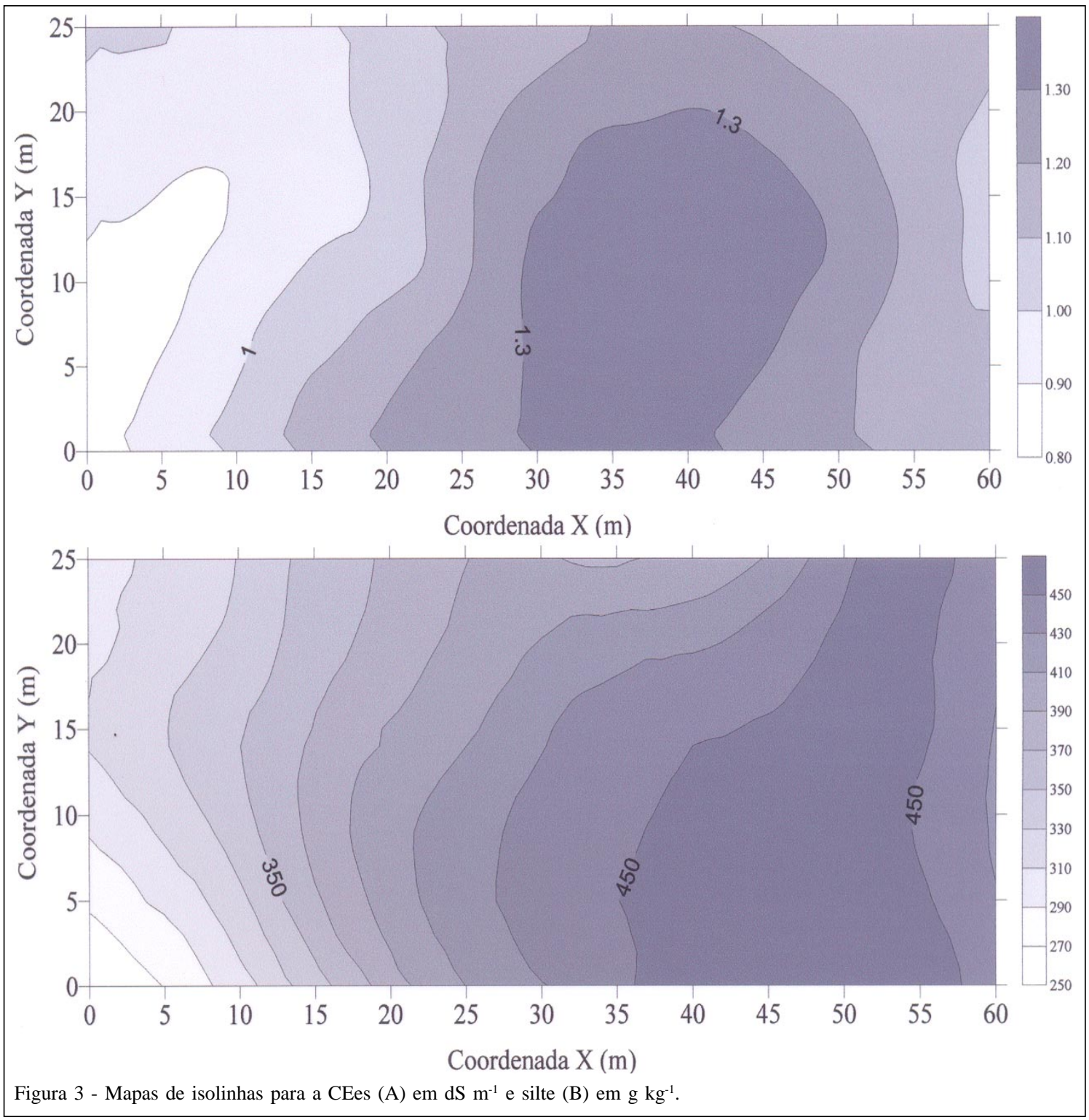

argila que se caracteriza como de baixa variabilidade. As frações granulométricas apresentam alta dependência espacial, com alcances de 56, 50 e 55m para areia, argila e silte respectivamente, enquanto que a condutividade elétrica do extrato de saturação apresenta dependência moderada. Por meio dos mapas de isolinhas, percebe-se que as áreas com maiores valores de CEes apresentaram teores elevados de silte, ressaltando a importância dessa fração para o manejo de solos francos.

\section{AGRADECIMENTOS}

Os autores agradecem o apoio do Conselho Naciona de Desenvolvimento Científico e Tecnológico (CNPq/CT-Hidro e CT-Agro), bem como a preciosa colaboração dos revisores.

\section{REFERÊNCIAS}

BASTOS, D.C.O. Manejo da salinidade em irrigação localizada: análise da alternativa de lixiviação incompleta. 2004. 91f. Dissertação (Mestrado em Ciência do Solo) - Curso de Pós-graduação em Ciência do Solo, Universidade Federal Rural de Pernambuco.

CAMBARDELLA, C.A. et al. Field scale variability of soil properties in Central Iowa soils. Soil Science Society of America Journal, Madison, v.58, n.5, p.1501-1511, 1994.

CICHOTA, R. et al. Variabilidade espacial da taxa de infiltração em argissolo vermelho. Revista Brasileira de Ciência do Solo, Viçosa, v.27, n.5, p.789-798, 2003.

COELHO, M.G. Variabilidade espacial de características físicas e químicas em um solo salino-sódico. Ciência Agronômica, Fortaleza, v.14, n.01/02, p.149-156, 1983. 
CRESSIE, N.A.; HAWKINS, D. Robust estimation of the variogram. Journal of International Association of Mathematical Geology, Kingston, v.12, n.1, p.115-125, 1980.

EGUCHI, E.S. et al. Variabilidade espacial da textura e da densidade de partículas em um solo aluvial no Município de Lavras, MG. Revista Brasileira de Engenharia Agrícola e Ambiental, Campina Grande, v.6, n.2, p.242-246, 2002.

EMPRESA BRASILEIRA DE PESQUISA AGROPECUÁRIA EMBRAPA. Manual de métodos de análise de solo. 2.ed. Rio de Janeiro: Ministério da Agricultura e do Abastecimento, 1997. 212p

ENGLUND, E.; SPARKS, A. Geo-EAS (Geostatistical Environmental Assessment Software). Las Vegas: U.S. Environmental Protection Agency, 1991. EPA/600/4-88/033 .

FAULIN, G.C. Variabilidade espacial do teor de água e sua influência na condutividade elétrica do solo. 2005. 52f. Dissertação (Mestrado em Agronomia) - Curso de Pósgraduação em Agronomia, Escola Superior de Agricultura Luiz de Queiroz.

GREGO, C.L.; VIEIRA, S.R. Variabilidade espacial de propriedades físicas do solo em uma parcela experimental. Revista Brasileira de Ciência do Solo, Viçosa, v.29, n.2, p.169-177, 2005.

HARGREAVES, G.H. Climatic zoning for agricultural production in northeast Brazil. Logan: Utah State University, 1974. 6p.

HOAGLIN, D.C. et al. Análisis exploratória de datos: técnicas robustas, un guia. Lisboa: Salamandra, 1983. 446p.

JOURnEL, A.G.; HUIJBREGTS, J. Mining geostatistics. England: Academic,. 1978. 599p.

MONTENEGRO, S.M.G.L. et al. Caracterização da variabilidade espacial de parâmetros hidráulicos em solos aluviais no estado de Pernambuco. Revista Brasileira de Recursos Hídricos, Porto Alegre, v.4, n.2, p.3-20, 1999.

MONTENEGRO. A.A.A.; MONTENEGRO, S.M.G.L. Variabilidade espacial de classes de textura, salinidade e condutividade hidráulica de solos em planície aluvial. Revista Brasileira de Engenharia Agrícola e Ambiental, v.10, n.1, p.30-37, 2006.

REICHARDT, K.; TIMM, L.C. Solo, planta e atmosfera: conceitos, processos e aplicações. Barueri, SP: Manole, 2004. 478p.

RIBEIRO, M.R. et al. Solos halomórficos no Brasil: Ocorrência, gênese, classificação, uso e manejo sustentável. In: CURI, N. et al. Tópicos em ciência do solo. Viçosa, Minas Gerais: Sociedade Brasileira de Ciência do Solo, 2003. v.III, 430p.

RICHARDS, L.A. Diagnosis and improvement of saline and alkali soils. Washington, D.C.: United States Salinity Laboratory, 1954. 160p. (United States Department of Agriculture - Handbook, 60).

SOUZA, L.C. et al. Variabilidade espacial da salinidade de um solo aluvial no semi-árido paraibano. Revista Brasileira de Engenharia Agrícola e Ambiental, Campina Grande, v.4, n.1, p.35-40, 2000.

SURFER. Surfer 8.0: contouring and $3^{\text {rd }}$ surface mapping for scientists and engineers. User's Guide. New York: Golden Software, 2002. 619p.

VAUCLIN, M. et al. The use of cokriging with limited field soil observations. Soil Science Society of America Journal, Madison, v.47, n.1, p.175-184, 1983.

VIEIRA, S.R. Variabilidade espacial de argila, silte e atributos químicos em uma parcela experimental em um latossolo roxo de Campinas (SP). Bragantia, Campinas, v.57, n.1, p.181190, 1997.

WARRICK, A.W.; NIELSEN, D.R. Spatial variability of soil physical properties in the field. In: HILLEL, D. Environmental soil physics. New York: Academic, 1998. p.655-675. 